\title{
KLF6 contributes to myeloid cell plasticity in the pathogenesis of intestinal inflammation
}

\author{
WA Goodman ${ }^{1}$, S Omenetti $^{1}$, D Date ${ }^{2}$, L Di Martino ${ }^{2}$, C De Salvo ${ }^{1}$, G-D Kim ${ }^{2}$, S Chowdhry ${ }^{2,3}$, G Bamias ${ }^{4}$, \\ F Cominelli $^{2,3}$, TT Pizarro ${ }^{1,2,5}$ and GH Mahabeleshwar ${ }^{1,2,5}$
}

Inflammatory bowel disease (IBD) is associated with dysregulated macrophage responses, such that quiescent macrophages acquire a pro-inflammatory activation state and contribute to chronic intestinal inflammation. The transcriptional events governing macrophage activation and gene expression in the context of chronic inflammation such as IBD remain incompletely understood. Here, we identify Kruppel-like transcription factor-6 (KLF6) as a critical regulator of pathogenic myeloid cell activation in human and experimental IBD. We found that KLF6 was significantly upregulated in myeloid cells and intestinal tissue from IBD patients and experimental models of IBD, particularly in actively inflamed regions of the colon. Using complementary gain- and loss-of-function studies, we observed that KLF6 promotes pro-inflammatory gene expression through enhancement of nuclear factor $\kappa B$ (NFKB) signaling, while simultaneously suppressing anti-inflammatory gene expression through repression of signal transducer and activator of transcription 3 (STAT3) signaling. To study the in vivo role of myeloid KLF6, we treated myeloid-specific KLF6-knockout mice (Mac-KLF6-KO) with dextran sulfate sodium (DSS) and found that Mac-KLF6-KO mice were protected against chemically-induced colitis; this highlights the central role of myeloid KLF6 in promoting intestinal inflammation.

Collectively, our results point to a novel gene regulatory program underlying pathogenic, pro-inflammatory macrophage activation in the setting of chronic intestinal inflammation.

\section{INTRODUCTION}

The human gastrointestinal tract is home to more than one hundred trillion commensal microbes, ${ }^{1}$ highlighting the need to discriminate between beneficial organisms and pathogenic, invading microbes. The innate immune system of the intestinal mucosa has therefore evolved to provide a rapid, first-line defense against harmful, infectious organisms through the recognition of conserved molecular patterns unique to invading pathogens, such as endotoxins and nucleic acids. Pattern recognition receptor-bearing macrophages situated in the subepithelial lamina propria of the small and large intestine are central to this process, and in fact represent the largest pool of tissue-resident macrophages in the body. ${ }^{2}$ In addition to their essential roles in maintaining host defense, scavenging dead cells and debris, and helping to maintain epithelial barrier function, ${ }^{3}$ intestinal mucosal macrophages are required for shaping immune responses to the continuous barrage of antigenic challenges that breach the intestinal epithelium.

The requirement of macrophages for maintenance of intestinal homeostasis was established by seminal work in which deletion of macrophages ${ }^{4,5}$ or toll-like receptors and their common signaling adapter, MyD88, ${ }^{6}$ resulted in severe morbidity and mortality in response to dextran sulfate sodium (DSS)-induced colitis. These studies suggest that under noninflamed conditions, intestinal macrophages recognize commensal microbes through pattern recognition receptor interactions and regulate mucosal immunity in response to these signals. Intestinal macrophages are also required for the development of mucosal tolerance, as macrophage-deficient mice (F4/80 knockout (KO)) fail to develop tolerance or

\footnotetext{
${ }^{1}$ Department of Pathology, Case Western Reserve University School of Medicine, Cleveland, Ohio, USA. ²Department of Medicine, Case Western Reserve University Schoo of Medicine, Cleveland, Ohio, USA. ${ }^{3}$ Department of Medicine, Division of Gastroenterology, University Hospitals of Cleveland, Cleveland, Ohio, USA and ${ }^{4}$ Academic Department of Gastroenterology, Ethnikon and Kapodistriakon University of Athens and Laikon Hospital, Athens, Greece. Correspondence: GH Mahabeleshwar or TT Pizarro (ghm4@case.edu or theresa.pizarro@case.edu)

${ }^{5}$ The last two authors contributed equally to this work.

Received 31 July 2015; accepted 29 November 2015; published online 3 February 2016. doi:10.1038/mi.2016.1
} 
antigen-specific regulatory $\mathrm{T}$ cells (Treg) following oral administration of soluble antigen. ${ }^{7}$

Innate immunity sensing mechanisms are critical for initiating and shaping the adaptive immune response in the lamina propria ${ }^{8,9}$ and other tissues, as demonstrated by the ability of "classically" (M1) and "alternatively" (M2) activated macrophages to promote Th1 and Th2 responses, respectively. Although M1/M2 macrophage classification remains widely used, these broad categories encompass many different phenotypic and functional subsets. ${ }^{10}$ Macrophages are differentiated and activated by many different stimuli, including cytokines, toll-like receptor agonists, and other exogenous stimuli. Responses to stimulation by these agents, alone or in combination, can lead to a spectrum of activation states not restricted to the classic M1/M2 classificiations. Recent efforts have therefore led to the development of a new consensus framework for the classification of activated macrophages, aimed at standardizing macrophage nomenclature. ${ }^{10}$

Under homeostatic conditions, intestinal macrophages remain "inflammation anergic" 11 in response to strong signals from anti-inflammatory interleukin (IL)-10 and TGF $\beta$ (transforming growth factor beta), displaying many phenotypic and functional features of M2 macrophages. Although these cells express pattern recognition receptors and display robust phagocytic activity, they fail to mount inflammatory responses to exogenous antigens; ${ }^{12}$ this unique feature allows intestinal macrophages to effectively eliminate foreign invaders while simultaneously maintaining immune quiescence. Myeloid-specific deletion of M2-associated molecules such as IL-10Ra, ${ }^{13}$ signal transducer and activator of transcription 3 (STAT3), ${ }^{14}$ and peroxisome proliferator-activated receptor gamma $(\operatorname{PPAR} \gamma)^{15}$ leads to a significant exacerbation of experimental colitis, highlighting the immunoprotective role conferred by these molecules under intestinal homeostasis.

In contrast, intestinal macrophages of inflammatory bowel disease (IBD) patients display an impairment in inflammatory anergy and an increase in pro-inflammatory, M1 activation. ${ }^{16}$ This process is thought to occur due to high levels of cytokines, such as interferon gamma (IFN $\gamma$ ), tumor necrosis factor (TNF), IL-1, and IL-6, in the gut mucosa that can divert infiltrating monocytes towards a pathogenic, M1 macrophage phenotype. ${ }^{3}$ These cells exhibit increased activation of nuclear factor $\kappa \mathrm{B}$ (NFkB), enhanced sensitivity to toll-like receptor ligands, and elevated expression of pro-inflammatory cytokines, chemokines, matrix metalloproteinases, and reactive oxygen species /nitric oxide synthases, ${ }^{17-19}$ leading to inappropriate inflammatory responses directed against non-harmful commensal microbes and dietary antigens. Blockade of macrophage-derived TNF is a standard therapeutic approach in the treatment of $\mathrm{IBD},{ }^{20}$ demonstrating the pathogenic contribution of inflammatory macrophages to IBD. Experimental IBD is also dependent on pathogenic macrophages and their cytokine products, as both depletion of macrophages directly $^{21}$ and treatment with anti-TNF antibodies ${ }^{22}$ are sufficient to suppress the development of spontaneous colitis in IL-10 $\mathrm{KO}$ mice.
Mature macrophages remain transcriptionally dynamic and can alter their activation state in response to environmental cues such as cytokines and other soluble mediators. Changes to the IBD cytokine environment, such as an upregulation of IFN $\gamma$, TNF, IL-1, and IL-6, may therefore divert macrophages away from inflammatory anergy and toward a pathogenic, M1 phenotype. Given the plasticity of macrophage phenotypes and functions, it is critical to identify molecular events governing the loss of inflammatory anergy and the "switch" to pathogenic, M1 phenotypes that occurs in the setting of chronical intestinal inflammation such as IBD.

Kruppel-like transcription factors (KLFs) are a subclass of zinc-finger family transcription factors that regulate critical cellular processes, including development, differentiation, proliferation, and programmed cell death. ${ }^{23-25}$ Previous studies have indicated that KLF2 (ref. 26) and KLF4 (ref. 27) have important roles in macrophage inflammatory gene expression and function; however, it has been recently shown that KLF6 is the most highly expressed KLF family member in primary macrophages, where it simultaneously promotes M1 activation and inhibits M2 activation. ${ }^{28}$ KLF6 has also been recently linked to the pathogenesis of human inflammatory diseases, including psoriasis ${ }^{29}$ and hepatic steatosis. ${ }^{30}$ Nonetheless, the role of myeloid-derived KLFs, including KLF6, in the pathogenesis of IBD has never been investigated.

Our current studies seek to investigate the contribution of macrophage KLF6 to the loss of macrophage inflammatory anergy in IBD. We demonstrate a significant upregulation of KLF6 in intestinal myeloid cells from human and experimental IBD subjects, as well as a central role for KLF6 in controlling macrophage responses to pro- and anti-inflammatory stimuli associated with the development and resolution of intestinal inflammation. Thus, we propose that KLF6 may function as a molecular switch, diverting intestinal macrophages away from protective, inflammatory anergic phenotypes and toward pathogenic, M1 phenotypes in response to the local IBDassociated cytokine milieu.

\section{RESULTS}

\section{Myeloid KLF6 is upregulated in human and experimental} IBD

Our previous work demonstrated that KLF6 is the most highly expressed Kruppel-like transcription factor in macrophages and contributes to pro-inflammatory macrophage activation and function. ${ }^{28}$ Given the role of activated macrophages in human and experimental IBD, ${ }^{12}$ we sought to investigate whether myeloid cell KLF6 expression is associated with intestinal inflammation. Analysis of mRNA isolated from intestinal biopsies of ulcerative colitis (UC) and Crohn's colitis disease (CD) patients, two of the primary idiopathic forms of IBD, revealed elevated KLF6 gene expression within both patient cohorts compared with intestinal tissues from noninflamed, healthy controls (Figure 1a). To determine whether higher levels of KLF6 correlate with worsened clinical disease, we obtained paired biopsy samples from uninflamed regions 
a

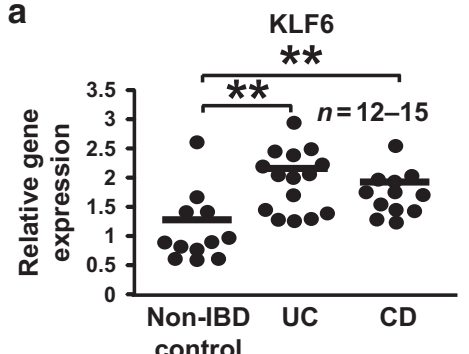

d

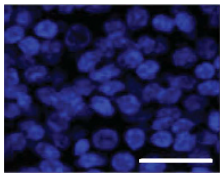

Draq-5

(nuclear)

e

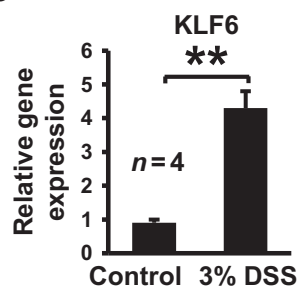

f

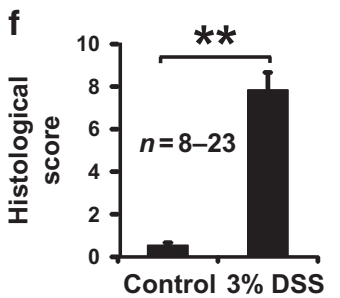

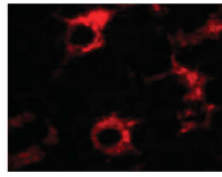

$\alpha$-CD68 (Alexa594)

g
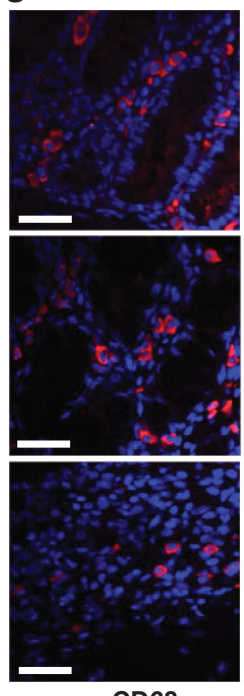

$\alpha-C D 68$

(Alexa-594)
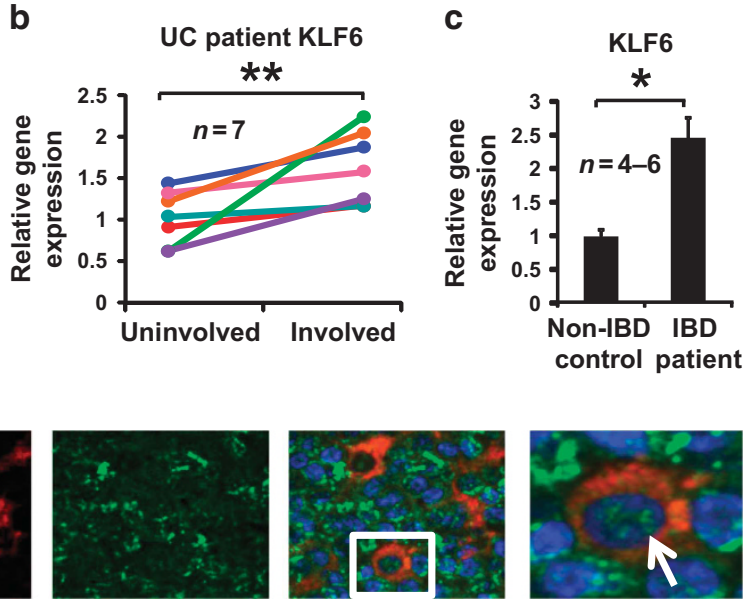

$\alpha$-KLF6 (Alexa488)

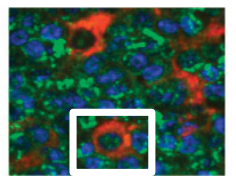

Merged

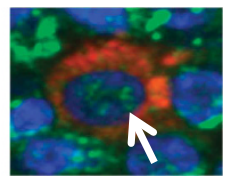

Merged (300\%)
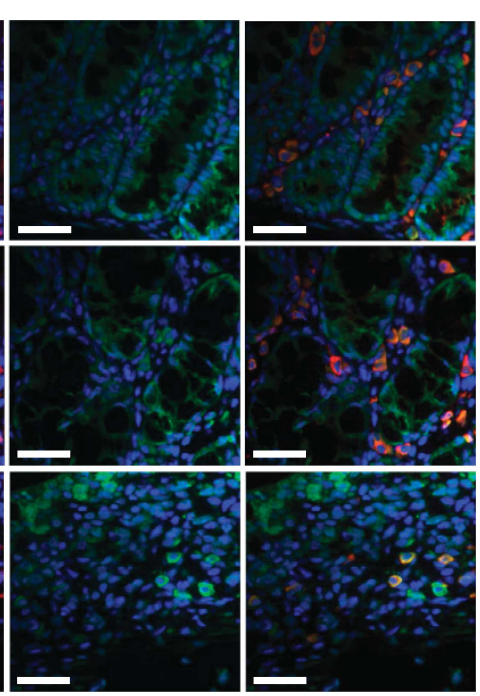

$\alpha-$ KLF6

(Alexa-488)

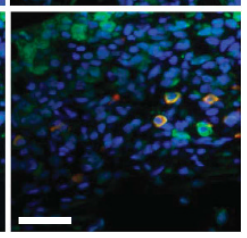

Merged
No DSS

control

\section{$3 \%$ DSS}

(mild/moderate)

Figure 1 Kruppel-like transcription factor-6 (KLF6) is upregulated in inflamed intestinal tissues and macrophages from human and experimental inflammatory bowel disease (IBD) subjects. (a) Colon tissues from non-IBD control subjects or ulcerative colitis (UC) or Crohn's colitis disease (CD) patients were homogenized, cDNA prepared, and analysis of KLF6 gene expression was performed by qPCR; ${ }^{* *} P \leqslant 0.001, n=12-15 / \mathrm{group}$. (b) Paired biopsies from uninvolved and involved regions of UC patient colon tissue were analyzed for KLF6 gene expression by qPCR. Fold changes in involved samples were calculated relative to uninvolved (baseline) samples and normalized to expression of $S H D A$. Lines represent individual patients; ${ }^{* \star} P \leqslant 0.03$, $n=7$. (c) Monocyte-derived macrophages from non-IBD control subjects or IBD patients were analyzed for $K L F 6$ gene expression by qPCR; ${ }^{*} P \leqslant 0.01$, $n=4-6 /$ group. (d) KLF6-expressing myeloid cells were detected in human mesenteric lymph nodes by immunofluorescent confocal microscopy. Formalin-fixed, paraffin-embedded mesenteric lymph-node tissues were stained with antibodies specific to CD68 (myeloid cell marker, imaged with Alexa 594 as red) and KLF6 (imaged with Alexa 488 as green). Nuclei are stained with Draq5 (imaged as blue); $n=3$ independent experiments. Scale $\mathrm{bar}=100 \mu \mathrm{m}$; white arrow indicates representative KLF6-expressing macrophage. (e) Macrophages from $3 \%$ dextran sulfate sodium (DSS)-fed mice express elevated levels of KIf6. Peripheral blood-derived macrophages from wild-type (WT) mice were analyzed for KIf6 gene expression by qPCR; Graphs show mean \pm s.e.m.; ${ }^{* \star} P \leqslant 0.001, n=4$ /group. (f) Histological colon inflammation was assessed by a blinded pathologist following 5 days of $3 \%$ DSS. Score consists of active, chronic, and transmural inflammation; ${ }^{* \star} P \leqslant 0.001, n=8-23$ mice/group. (g) KLF6-expressing myeloid cells are observed in colon tissue from DSS-fed mice by confocal microscopy. Formalin-fixed, paraffin-embedded colon tissues from indicated mice were stained by immunofluorescent confocal microscopy using antibodies specific to CD68 and KLF6, as described above (c). Nuclei were stained with Draq5. Scale $\mathrm{bar}=34 \mu \mathrm{m}$. Images are representative of three separate experiments.

("uninvolved") and acutely inflamed colon tissue regions ("involved") from CD and UC patients. Although comparable levels of KLF6 mRNA were detected in involved and uninvolved samples from CD patients (Supplementary Figure S1 online), significantly higher levels of KLF6 were detected in involved regions of UC patient colons, compared with uninvolved regions from the same individuals (Figure 1b). Monocytederived macrophages from IBD patients also revealed a significant upregulation in KLF6 mRNA compared with macrophages from healthy controls (Figure 1c). 
To determine whether myeloid KLF6 is predominantly nuclear or cytoplasmic, we performed immunofluorescent staining on mesenteric lymph node tissue sections obtained from IBD patients undergoing surgical resection. Abundant KLF6 was observed throughout the mesenteric lymph node (green, Figure 1d) in the cytoplasm and nuclei of $\mathrm{CD} 68^{+}$ myeloid cells (red, Figure 1d) as well as lymphocytic cells, demonstrating that numerous cell populations in the intestine express KLF6.

To determine whether these observations are recapitulated in experimental models of IBD, we assessed gene expression of Klf6 in standard models of ileitis $\left(\mathrm{TNF}^{\mathrm{ARE} /+}\right)^{31}$ and colitis (DSS-fed mice). ${ }^{32}$ Monocyte-derived macrophages from DSS colitic mice (Figure 1e) and ileal tissues from $\mathrm{TNF}^{\Delta \mathrm{ARE} /+}$ mice (Supplementary Figure S2) revealed significantly elevated levels of Klf6 mRNA in diseased tissue/macrophages compared with non-diseased control tissues and cells. Histological examination of colon tissues from DSS-treated, wild-type (WT) mice revealed characteristic features of inflammation, including epithelial erosion, ulceration, immune cell infiltration, and edema, and were quantified by an established histological scoring system (Figure 1f). ${ }^{33}$ Immunofluorescent confocal staining of colon tissues from control (no DSS)-fed mice and DSS-fed mice revealed prominent areas of $\mathrm{CD} 68^{+}$ myeloid cell infiltration in healthy, as well as inflamed, colons (Figure 1g, red). KLF6 was detected diffusely in epithelial cells in both control and DSS-fed mice (Figure 1g, green), although the intensity of epithelial KLF6 staining was greater in more severe regions of DSS-fed colon tissue (lower panels, Figure 1g). Interestingly, $\mathrm{CD}^{+} 8^{+}$myeloid cells expressed the highest levels of KLF6 in severely inflamed, DSS-fed colon tissue, and nearly all $\mathrm{CD}^{+} 8^{+}$cells in these mice expressed robust KLF6 staining. In contrast, KLF6 expression was weak or absent in the majority of $\mathrm{CD} 68^{+}$cells in non-DSS treated control mice, as well as less inflamed areas of DSS-fed mice (upper and middle panels). Collectively, these data indicate that elevated levels of myeloid KLF6 correlate with intestinal inflammation and tissue damage in IBD patients and experimental models, particularly in inflamed colitis patients and mice.

\section{KLF6 enhances the expression of IBD-associated pro-inflammatory genes in macrophages}

Using complementary gain- and loss-of-function approaches, we sought to test how modulation of KLF6 may influence myeloid cell responses to IFN $\gamma$, a pro-inflammatory cytokine central to the pathogenesis of IBD and inducer of M1 macrophage activation. To examine the effects of KLF6 overexpression, the RAW264.7 macrophage cell line was transiently transfected with a KLF6-expressing plasmid (pCI-neo-KLF6, Addgene, Cambridge, MA) or empty vector (EV) control, as previously described. ${ }^{28}$ Transfected cells were treated with phosphate-buffered saline (PBS) (Ctrl) or IFN $\gamma$, then analyzed for Tnf, Mcp1, inos, and $M c p 3$ gene expression. Macrophage overexpression of KLF6 resulted in a robust and significant upregulation of all four pro-inflammatory genes in response to IFN $\gamma$, compared with EV-transfected cells (Figure 2a). a

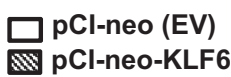

$n=5$
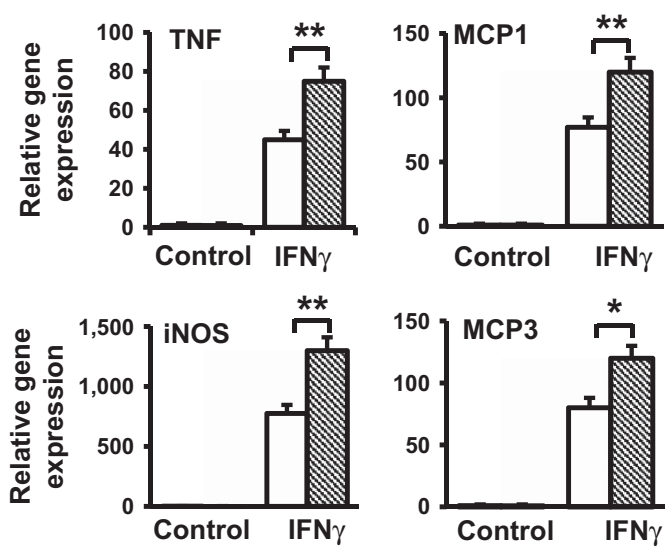

b
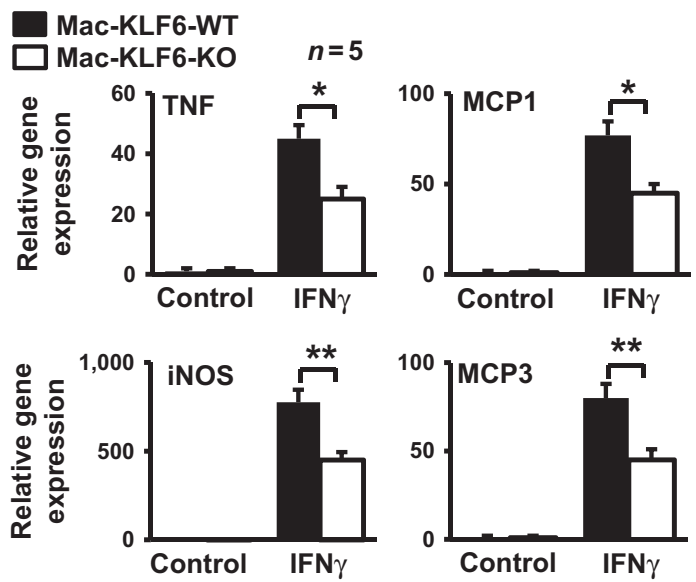

Figure 2 Kruppel-like transcription factor-6 (KLF6) induces proinflammatory gene expression in macrophages. (a) Macrophages overexpressing KLF6 show enhanced expression of pro-inflammatory genes. RAW264.7 macrophages were transfected with pCl-neo (empty vector (EV) plasmid control) or pCl-KLF6 to overexpress KLF6. Cells were stimulated in vitro with phosphate-buffered saline (PBS) (control) or interferon gamma (IFN $\gamma)\left(100 \mathrm{U} \mathrm{ml}^{-1}\right)$ for $6 \mathrm{~h}$, then harvested for RNA isolation. cDNA was analyzed for expression of pro-inflammatory genes Tnf, Mcp1, inos, and Mcp3 by qPCR; ${ }^{*} P \leqslant 0.02 ;{ }^{\star \star} P \leqslant 0.003 ; n=5 /$ group. (b) Macrophages deficient in KLF6 show reduced expression of proinflammatory genes. Peritoneal macrophages were isolated from MacKLF6-WT and Mac-KLF6-KO mice following thioglycolate elicitation. Cells were stimulated in vitro with PBS (control) or IFN $\gamma\left(100 \mathrm{U} \mathrm{ml}^{-1}\right)$ for $6 \mathrm{~h}$, then harvested for RNA isolation. cDNA was analyzed for expression of pro-inflammatory genes; ${ }^{\star} P \leqslant 0.03 ;{ }^{\star \star} P \leqslant 0.001, n=5 /$ group. All graphs show mean \pm s.e.m. iNOS, inducible nitric oxide synthase; KO, knockout; MCP, monocyte chemotactic protein; TNF, tumor necrosis factor; WT, wild type.

To evaluate the effects of KLF6 loss-of-function, we utilized myeloid-specific KLF6-null mice (Mac-KLF6-KO). These mice are generated by breeding Lyz2cre-expressing mice with KLF6 floxed mice, and display a robust reduction of KLF6 in primary macrophages. ${ }^{28}$ Littermate control mice carrying only the Lyz2cre gene (Mac-KLF6-WT) were used as controls for all experiments. Neither Mac-KLF6-WT nor Mac-KLF6-KO mice display any spontaneous, phenotypic features of IBD. Using thioglycolate-elicited peritoneal macrophages (PMs) from 
Mac-KLF6-KO mice and littermate controls (Mac-KLF6-WT), we tested whether KLF6 expression is essential for IFN $\gamma$ induced inflammatory gene expression in macrophages. In agreement with our overexpression studies (Figure 2a), deficiency of KLF6 significantly attenuated IFN $\gamma$-induced Tnf, $M c p 1$, inos, and $M c p 3$ gene expression in macrophages (Figure $2 \mathbf{b}$ ). Taken together, these results implicate a role for macrophage KLF6 in IBD-associated pro-inflammatory gene expression.

\section{KLF6 suppresses the expression of IBD-associated anti- inflammatory genes in macrophages}

Under homeostatic conditions, high levels of IL-10 retain intestinal macrophages in an anti-inflammatory state referred to as "inflammatory anergy". 11 To determine whether macrophage KLF6 actively inhibits IL-10-induced anti-inflammatory gene targets, we utilized gain- and loss-of function approaches. Accordingly, RAW264.7 cells overexpressing KLF6 (or EV control), as well as Mac-KLF6-KO and Mac-KLF6-WT PM, were stimulated with IL-10 and then harvested for mRNA isolation. Interestingly, we found that expression of IL-10induced anti-inflammatory genes such as Socs3, Bcl3, Sbno2, and Illra was reduced in RAW264.7 macrophages overexpressing KLF6 compared with EV-transfected controls (Figure 3a). Mac-KLF6-KO PM expressed higher levels of all four anti-inflammatory genes compared with Mac-KLF6WT PM in response to IL-10 (Figure 3b).

Given our observations of KLF6-mediated enhancement of IFN $\gamma$-induced pro-inflammatory gene expression and attenuation of IL-10-induced anti-inflammatory gene expression in RAW macrophages (Figures 2 and 3), we next examined whether pro- or anti-inflammatory cytokine stimulation affects macrophage KLF6 expression directly. Human monocytederived macrophages were stimulated with IFN $\gamma$, TNF, IL-10, or TGF $\beta$, harvested for mRNA and protein isolation, and assayed for KLF6 expression. IFN $\gamma$ and TNF stimulation of human macrophages resulted in an induction of Klf6 mRNA and protein (Supplementary Figure S3A), whereas IL-10 and TGF $\beta$ stimulation resulted in a downregulation of Klf6 mRNA and protein (Supplementary Figure S3B). Thus, pro- and anti-inflammatory cytokine signals can directly modulate the expression of KLF6 in human macrophages.

\section{Deficiency of myeloid KLF6 protects against experimental colitis}

Having established that KLF6 regulates, and is regulated by, inflammatory cytokine signaling (Figures 2 and 3), we next sought to test the in vivo, functional role of KLF6 in intestinal inflammation. We challenged Mac-KLF6-KO and Mac-KLF6WT mice with 3\% DSS. ${ }^{32}$ When fed 3\% DSS for 5 days and allowed no recovery time (acute model of colitis, with mice killed on day 6), Mac-KLF6-WT mice exhibited severe weight loss over time (approximately $11 \%$ from baseline, Figure 4a). In contrast, Mac-KLF6-KO mice were partially protected from DSS-induced weight loss, averaging only a $5 \%$ loss in body weight by day 6 (Figure $4 \mathbf{a}$ ).

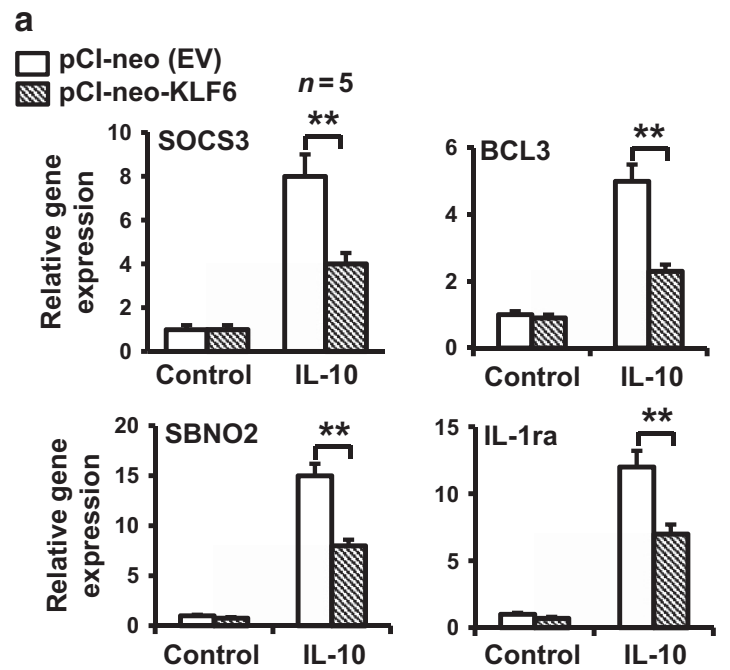

b
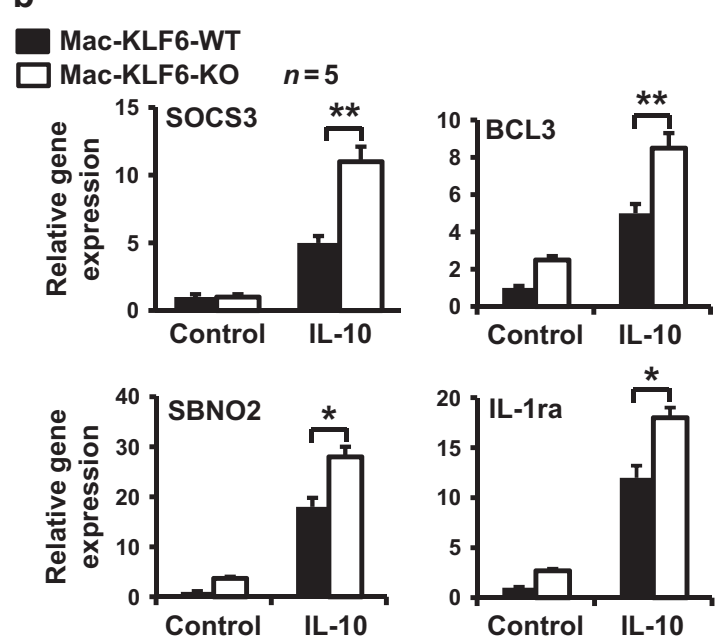

Figure 3 Kruppel-like transcription factor-6 (KLF6) inhibits antiinflammatory gene expression in macrophages. (a) Macrophages overexpressing KLF6 show reduced expression of anti-inflammatory genes. RAW264.7 macrophages were transfected with pCl-neo (empty vector (EV) plasmid control) or pCI-KLF6 to overexpress KLF6. Cells were stimulated in vitro with phosphate-buffered saline (PBS) (control) or interleukin (IL)-10 (10 $\mathrm{ng} \mathrm{ml}^{-1}$ ) for $18 \mathrm{~h}$, then harvested for RNA isolation. cDNA was analyzed for expression of anti-inflammatory genes Sosc3, $B c / 3$, Sbco2, and I/1ra by qPCR; ${ }^{\star} P \leqslant 0.03 ;{ }^{* \star} P \leqslant 0.001, n=5 /$ group. (b) Macrophages deficient in KLF6 show enhanced expression of antiinflammatory genes. Peritoneal macrophages were isolated from MacKLF6-WT and Mac-KLF6-KO mice following thioglycolate elicitation. Cells were stimulated in vitro with PBS (control) or IL-10 (10 $\mathrm{ng} \mathrm{ml}^{-1}$ ) for $18 \mathrm{~h}$, then harvested for RNA isolation. cDNA was analyzed for expression of anti-inflammatory genes. Graphs show mean \pm s.e.m.; * $P \leqslant 0.05$, ${ }^{\star \star} P \leqslant 0.01, n=5$ /group. BCL, B cell lymphoma 2; IL, interleukin; $\mathrm{KO}$, knockout; WT, wild type; SBNO, strawberry notch homolog; SOCS, suppressor of cytokine signaling.

Colon tissue from water-fed Mac-KLF6-WT and MacKLF6-KO mice appeared healthy and exhibited no phenotypic features of colitis (Figure $\mathbf{4 b}$, upper panels). In contrast, histological examination of colon tissue from Mac-KLF6-WT and Mac-KLF6-KO mice following DSS treatment revealed marked signs of inflammation in Mac-KLF6-WT compared with Mac-KLF6-KO (Figure 4b, lower panels). In agreement 


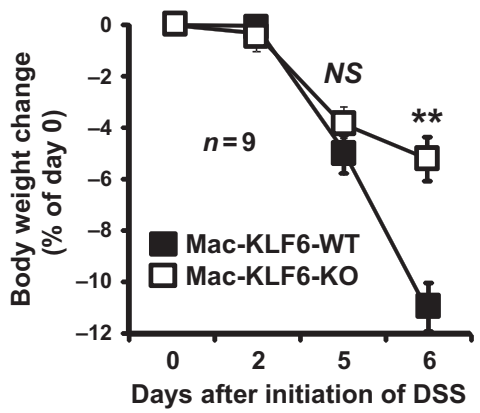

b
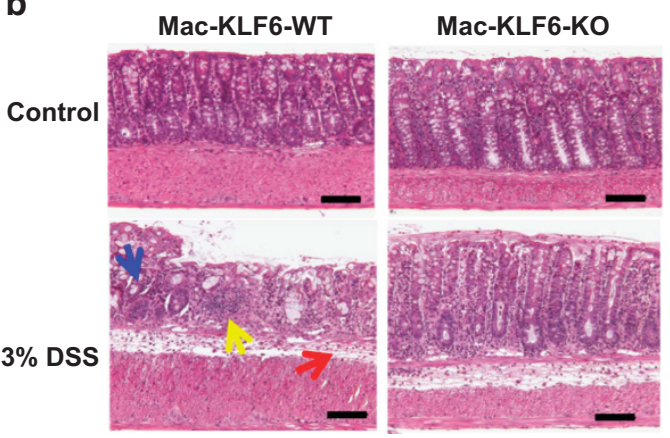

e

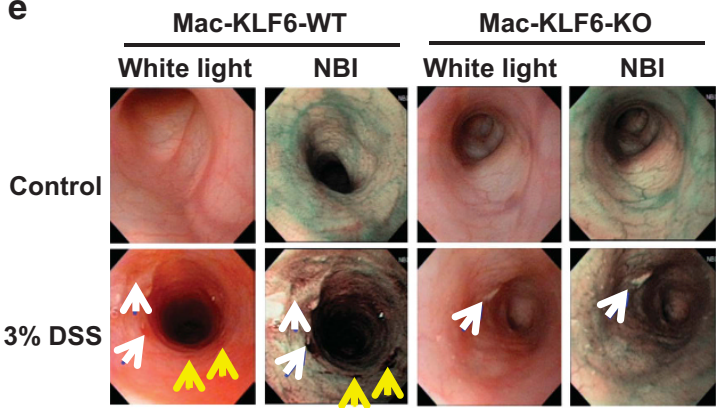

C

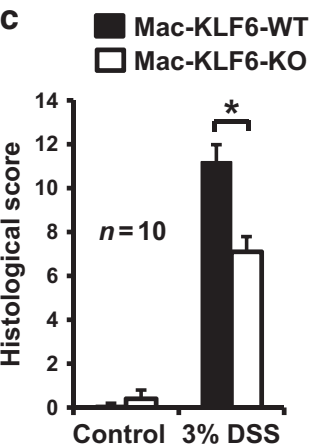

d

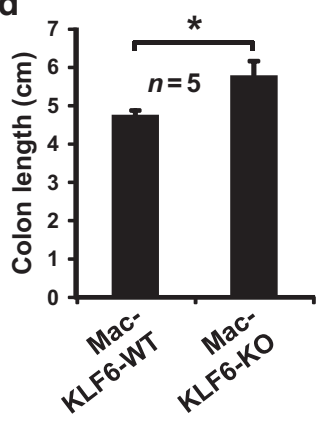

f Mac-KLF6-WT

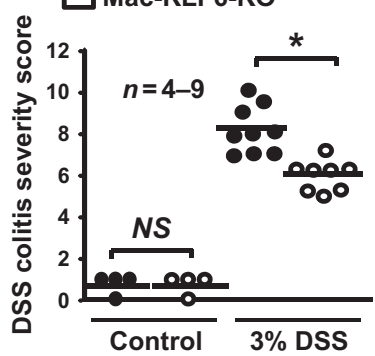

Figure 4 Myeloid deficiency of Kruppel-like transcription factor-6 (KLF6) is protective against dextran sulfate sodium (DSS)-induced colitis. Mac-KLF6KO and Mac-KLF6-WT mice were placed on $3 \%$ DSS for 5 days to induce colitis. (a) Body weight was measured on days $0,2,5$, and 6 , and is graphed relative to day 0 weight; ${ }^{* \star} P \leqslant 0.001, n=9$ mice/group. (b) Representative H\&E-stained colon sections from Mac-KLF6-KO and Mac-KLF6-WT mice following 5 days of $3 \%$ DSS. Arrows indicate disruption of epithelial architecture (blue); infiltration of immune cells (yellow), and edema (red); $n=10$ mice/ group. (c) Colon inflammation was assessed by a blinded pathologist following 5 days of $3 \%$ DSS. Score consists of active, chronic, and transmural inflammation; ${ }^{\star} P \leqslant 0.01, n=10$ mice/group. (d) Total colon length was measured following 5 days of $3 \% \mathrm{DSS} ;{ }^{*} P \leqslant 0.01, n=5$ mice/group. (e) Mice were subjected to colonic endoscopy following 5 days of $3 \%$ DSS. Representative images show endoscopy findings (left; arrows indicate colonic ulceration (white); mucus (blue); and erythematous lesions (yellow)) and perianal inflammation with diarrhea (right). Images are representative of 4-9 mice/group. (f) DSS colitis severity scores were assigned based on endoscopic and perianal findings. Graphs show mean \pm s.e.m. ${ }^{*} P \leqslant 0.03 ; n=8-9$ mice/group. KO, knockout; NBI, narrow-band imaging; NS, not significant; WT, wild type.

with histological observations, Mac-KLF6-WT mice exhibited significantly higher colitis scores than Mac-KLF6-KO mice (Figure 4c). We also evaluated colon length in Mac-KLF6-WT and Mac-KLF6-KO mice following DSS challenge, as colonic shortening is a well-established feature of acute colitis, and found that colons from Mac-KLF6-WT mice were significantly shortened compared with Mac-KLF6-KO colons following DSS administration (Figure 4d).

To examine colons in situ, we took advantage of a novel flexible colonoscopy procedure recently developed in our laboratory. ${ }^{34}$ This technique utilizes both narrow-band imaging and white light imaging, allowing for enhanced detection of erythematous regions and blood vessels (narrow-band imaging) in addition to visualization of mucus and ulcerated regions. The procedure was well tolerated and revealed significant colonic inflammation in Mac-KLF6-WT mice (Figure 4e), including ulcerated tissue (white arrows) and erythematous regions (yellow arrows). In contrast, colon imaging of MacKLF6-KO mice revealed only minimal ulceration and erythema (Figure 4e). Features of colitis observed by endoscopy were converted to decimal scores encompassing four parameters: perianal findings; wall transparency; intestinal bleeding; and focal lesions (edematous areas/erosions/ulcers). This quantitative endoscopic evaluation (Figure 4f) revealed 
significantly worse disease in Mac-KLF6-WT mice than in Mac-KLF6-KO mice, further implicating a pathogenic contribution for KLF6 to intestinal inflammation. Mac-KLF6-KO and Mac-KLF6-WT mice fed water, in the absence of DSS, showed no detectable signs of colitis by endoscopy (Figure 4e, upper panels).

\section{Myeloid KLF6 modulates inflammatory gene expression in colon tissue}

To determine effects of myeloid KLF6 deficiency on gene expression in the inflamed intestine, colon tissues were harvested from Mac-KLF6-KO and Mac-KLF6-WT mice following acute (5 days) DSS challenge. Tissues were analyzed for expression of pro- and anti-inflammatory genes known to be altered in acute colitis. Following 5 days of DSS feeding, colon tissues from both Mac-KLF6-WT and Mac-KLF6-KO mice expressed significantly elevated mRNA specific for the pro-inflammatory genes, Illa, Il1b, Il6, Tnf, inos, Mip1a, Mip1b, Il33, and Mcp1, compared with non-DSS-fed control mice (Figure 5a). However, DSS-fed Mac-KLF6-WT mice expressed significantly heightened expression of these genes compared with Mac-KLF6-KO mice (Figure 5a), in agreement with exacerbated disease in Mac-KLF6-WT mice (Figure 4). We also analyzed the expression of anti-inflammatory genes Arg1 and Il10, known to be protective in experimental and human intestinal inflammation. ${ }^{35}$ As expected, given their enhanced morphological and histological colitis phenotypes, Mac-KLF6WT mice displayed diminished expression of Arg1 and Il10 compared with Mac-KLF6-KO mice following acute DSS challenge (Figure 5b). Taken together, our results indicate that deficiency of myeloid KLF6 both diminishes DSS-induced pro-inflammatory gene expression and enhances antiinflammatory gene expression in colon tissues.

\section{KLF6 regulates macrophage inflammatory gene expression by modulating STAT3 and NFKB signaling pathways}

Our in vivo gene expression analysis indicated that myeloid deficiency of KLF6 enhanced the expression of Il10 in colon tissues (Figure 5b). Many IL-10-induced anti-inflammatory genes are transcriptionally regulated by STAT3. ${ }^{36}$ To test whether KLF6 overexpression and/or deficiency regulates IL-10-induced STAT3 transcriptional activity, we performed gain- and loss-of-function assays. Accordingly, RAW264.7 macrophages were co-transfected with STAT3 luciferase reporter plasmid (pTL-STAT3-Luc) in the presence of pCI-neo-KLF6 or siKLF6, stimulated with IL-10, and luciferase activity was measured. Overexpression of KLF6 attenuated (Figure 6a), whereas deficiency enhanced (Figure 6b), IL-10-induced, STAT3-dependent luciferase activity in RAW264.7 macrophages.

Our in vitro and ex vivo analyses indicate that KLF6 attenuates the expression of IL-10-induced, STAT3-regulated anti-inflammatory factors, such as suppressor of cytokine signaling 3 (SOCS3), in RAW264.7 macrophages (Figures 3a,b). We therefore evaluated whether KLF6 directly occupies the Socs3 gene promoter to repress its transcriptional activation. Results from chromatin immunoprecipitation (ChIP) assays using WT PMs indicate that KLF6 occupies the Socs3 promoter $(-313$ to -319$)$ under unstimulated conditions and that IL-10 exposure significantly diminishes KLF6 occupancy (Figure 6c). These findings indicate that KLF6 directly binds to STAT3 transcriptional targets, such as Socs3, to repress their expression. Deficiency of KLF6 results in a loss of this negative regulation, thereby enhancing expression of Socs 3 following IL-10 exposure (Figure 6c).

Next, we investigated whether the upregulation of IL-10induced anti-inflammatory genes in KLF6-deficient myeloid cells (Figure 3b) is STAT3 dependent. Mac-KLF6-WT and Mac-KLF6-KO PMs were stimulated with IL-10 in either the presence or the absence of the STAT3-specific inhibitor, NSC74859. As shown in Figure 6d, IL-10 exposure significantly elevated the STAT3 target gene Socs3 in MacKLF6-WT PMs. KLF6 deficiency further elevated IL-10induced Socs3 expression in myeloid cells. Interestingly, treatment with NSC74859 significantly attenuated IL-10induced Socs3 expression and eliminated the enhanced expression of Socs3 observed in KLF6-deficient myeloid cells. Taken together, our results indicate that KLF6 directly represses IL-10-induced anti-inflammatory gene expression by negatively regulating STAT3 transcriptional activity in myeloid cells.

Our in vitro and ex vivo analyses following IFN $\gamma$ exposure indicated that KLF6 enhances pro-inflammatory gene expression in RAW264.7 macrophages (Figure 2a,b). Therefore, RAW264.7 macrophages were co-transfected with an NFкB luciferase reporter plasmid in the presence of pCI-neo-KLF6 or siKLF6, stimulated with IFN $\gamma$, and luciferase activity was measured. Our results indicate that KLF6 overexpression significantly enhances (Figure 6e), while deficiency attenuates (Figure 6f), IFN $\gamma$-induced, NFKB-mediated luciferase activity in macrophages. Next, we evaluated whether KLF6 regulates the gene expression of classical NFKB pro-inflammatory targets, such as $M c p 1$, by directly binding to its promoter region. Our ChIP results indicate that KLF6 occupies the Mcp1 promoter $(-1,363$ to $-1,378)$ of WT PMs under unstimulated conditions, and that IFN $\gamma$ exposure results in enrichment of KLF6 by at least sixfold compared with controltreated cells (Figure 6g). Therefore, we hypothesized that KLF6 cooperates with $\mathrm{NF \kappa B}$ to regulate IFN $\gamma$-induced Mcp1 expression in RAW264.7 macrophages. Our results indicate that overexpression of either KLF6 or NFKB-p65 enhances IFN $\gamma$-induced monocyte chemotactic protein 1 (MCP1) expression compared with the pCI-neo-transfected control group (Figure $\mathbf{6 h}$ ). Importantly, cells stimulated with IFN $\gamma$ following combined overexpression of KLF6 and NFкB-p65 robustly induced $M c p 1$ expression compared with any other group, suggesting that KLF6 cooperates with NFKB to enhance IFN $\gamma$-induced Mcp1 expression in RAW264.7 macrophages. Taken together, our results clearly indicate that KLF6 promotes IFN $\gamma$-induced NFкB transcriptional activity and cooperates with $\mathrm{NF \kappa B}$ to regulate pro-inflammatory gene expression in myeloid cells. 
a
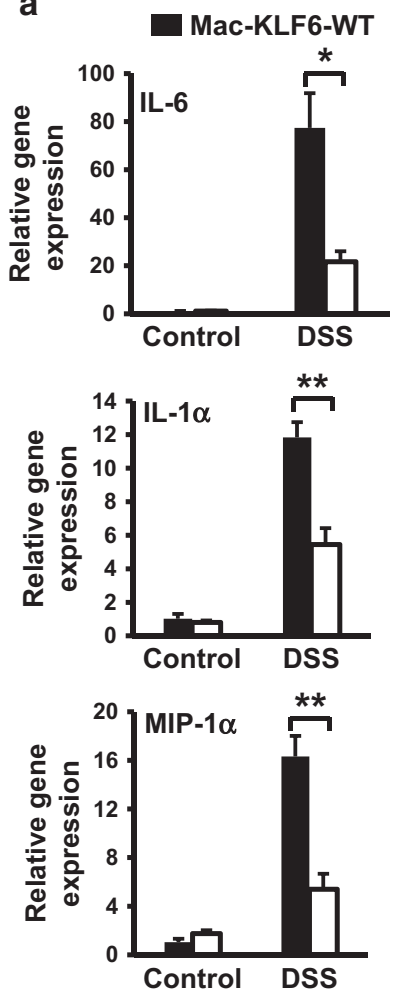

b

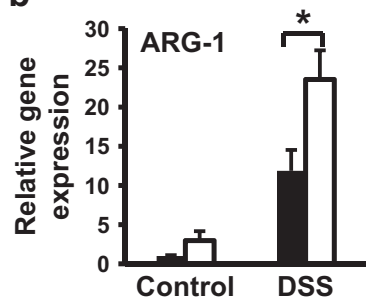

$\square$ Mac-KLF6-KO
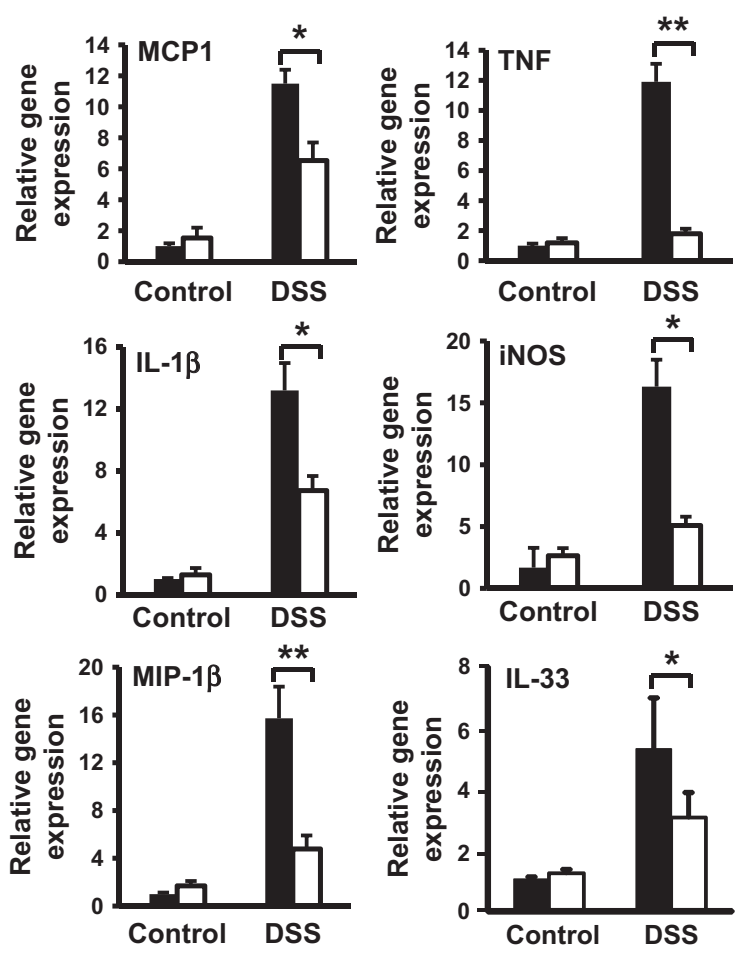

Figure 5 Myeloid Kruppel-like transcription factor-6 (KLF6) modulates inflammatory gene expression in colon tissue. Colon tissue from Mac-KLF6-WT and Mac-KLF6-KO mice was collected following 5 days of $3 \%$ dextran sulfate sodium (DSS) challenge (or water-fed control) and analyzed for gene expression. (a) cDNA was analyzed for expression of pro-inflammatory genes I/1a, I/1b, II6, Tnf, Mcp1, inos, Mip1a, Mip1b, and I/33. Gene expression was normalized to the housekeeping gene $36 \mathrm{~b} 4$, and is expressed as fold change of the control (water-fed) group; ${ }^{*} P \leqslant 0.01,{ }^{* *} P \leqslant 0.001, n=4-5 /$ group. (b) CDNA was analyzed for expression of anti-inflammatory genes Arg1 and I/10, then normalized to $36 \mathrm{~b} 4$ and expressed as fold change of the control group. Graphs show mean \pm s.e.m.; ${ }^{\star} P \leqslant 0.02$; ${ }^{\star *} P \leqslant 0.003, n=4-5 /$ group. IL, interleukin; iNOS, inducible nitric oxide synthase; KO, knockout; MIP, macrophage inflammatory protein; MCP, monocyte chemotactic protein; TNF, tumor necrosis factor; WT, wild type.

\section{DISCUSSION}

Our findings are the first to identify KLF6 as a significant transcriptional regulator in the context of intestinal inflammation, where it contributes to inflammatory macrophage activation and escape from inflammatory anergy. The key observations of this study are (i) KLF6 expression is elevated in diseased, colonic tissues, and myeloid cells from IBD patients, and high KLF6 levels correlate with acutely involved areas of UC colon tissue; (ii) experimental mouse models of IBD, including DSS-induced colitis and the $\mathrm{TNF}^{\triangle \mathrm{ARE} /+}$ model, reproduce the elevated KLF6 expression observed in IBD patients; (iii) macrophage KLF6 promotes IFN $\gamma$-induced proinflammatory gene regulatory programs and diminishes IL-10induced anti-inflammatory programs; (iv) in vivo deletion of myeloid-specific KLF6 partially protects against DSS-induced colitis, suggesting that endogenous myeloid KLF6 contributes to macrophage pro-inflammatory functions and exacerbates intestinal inflammation; and (v) the mechanisms by which KLF6 regulates macrophage phenotype and function are two-pronged, acting to enhance NFKB-mediated gene expression as well as to inhibit STAT3-mediated expression of anti-inflammatory genes. Collectively, our findings indicate that KLF6 contributes to transcriptional re-programming of intestinal macrophages in response to cytokine signals in the inflamed IBD intestine.

Macrophages are well known to alter their activation states in response to changing environmental stimuli. ${ }^{37}$ The spectrum of macrophage phenotypes and functions ranges from inflammatory anergic and related M2 macrophages, which promote tissue healing and repair processes, to pro-inflammatory M1 

activity

pCl-neo

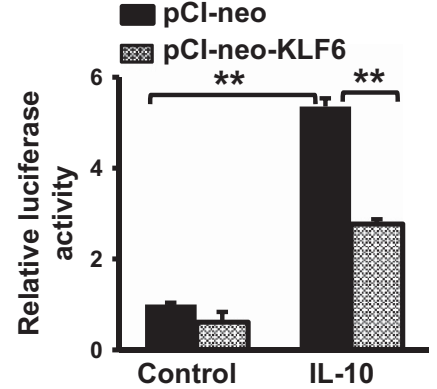

STAT3 luciferase reporter activity

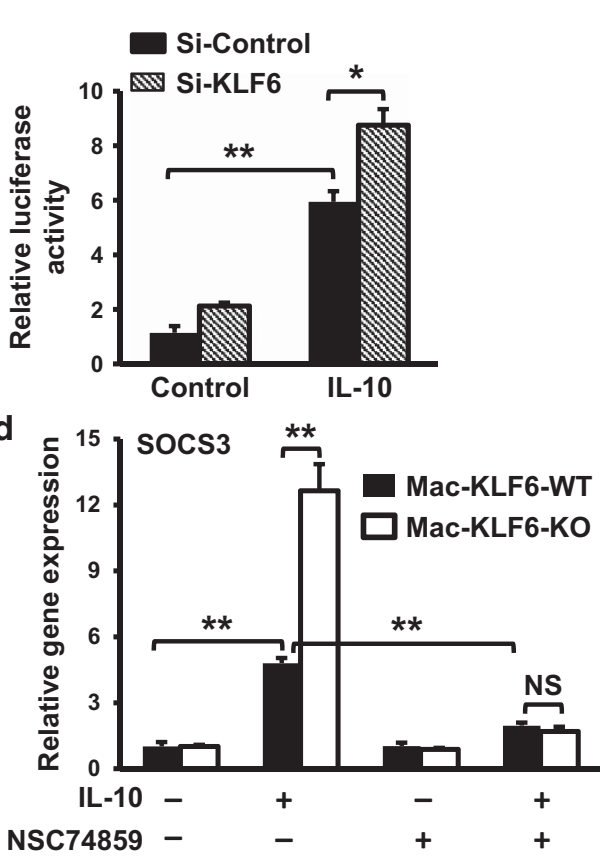

e NFkB concatemer luciferase

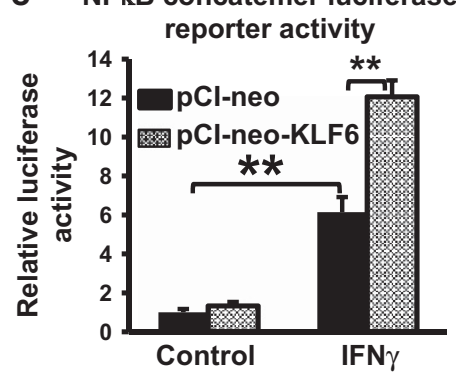

f NFkB concatemer luciferase

C socs3 promoter occupancy
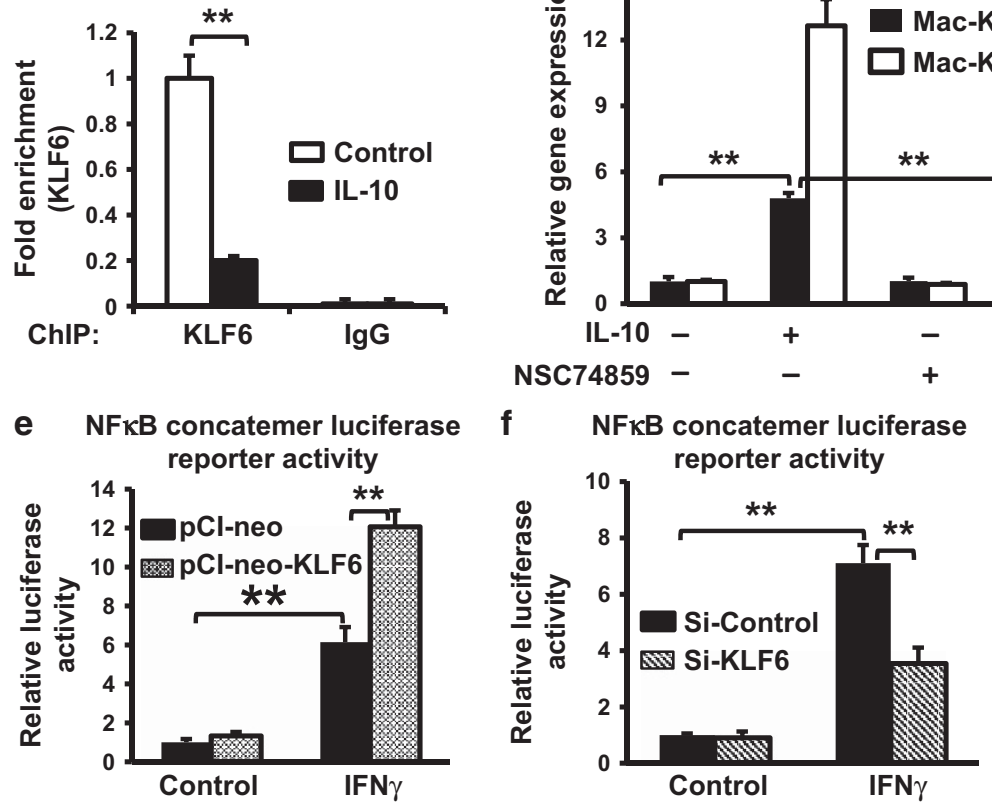

g MCP1 promoter occupancy

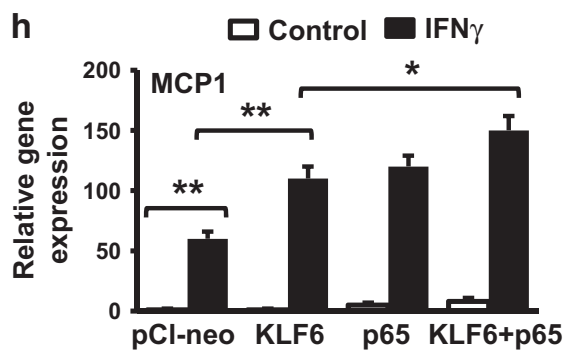

Figure 6 Kruppel-like transcription factor-6 (KLF6) interacts with pro-inflammatory and anti-inflammatory transcriptional machinery to regulate gene expression in response to cytokine signaling. (a) RAW264.7 macrophages were transfected to overexpress KLF6 (pCl-neo-KLF6) or control plasmid (pCl-neo) together with a signal transducer and activator of transcription 3 (STAT3) luciferase reporter plasmid. Cells were treated with interleukin (IL)-10 or phosphate-buffered saline (PBS) (control), then assessed for the relative luciferase activity, which is expressed as a ratio of control-transfected cells. (b) RAW264.7 macrophages were transfected with siRNA specific to KLF6 or control (scrambled) RNA, together with a STAT3 luciferase reporter plasmid. Cells were treated with IL-10 or PBS (Ctrl) and then assessed for luciferase activity. (c) Wild-type peritoneal macrophages (PMs) were treated with IL-10 or PBS (Ctrl), and chromatin immunoprecipitation (ChIP) assays were performed on the strawberry notch homolog 3 (SOCS3) promoter ( - 313 to -319) following immunoprecipitation with anti-KLF6 or IgG (Ctrl). (d) PMs from indicated mice were treated with IL-10 and/or the STAT3 inhibitor NSC74859 as indicated. Gene expression of SOCS3 was determined by QPCR, and is expressed as fold change over PBS-treated, wild-type PMs. (e) RAW264.7 macrophages were transfected to overexpress KLF6 (pCl-neo-KLF6) or control plasmid (pCl-neo) together with an nuclear factor $\kappa B$ (NFKB) luciferase reporter plasmid. Cells were treated with IFN $\gamma$ or PBS (control), then assessed for the relative luciferase activity. (f) RAW264.7 macrophages were transfected with siRNA specific to KLF6 or control (scrambled) RNA, together with an NFKB luciferase reporter plasmid. Cells were treated with IFN $\gamma$ or PBS (Control) and then assessed for luciferase activity. (g) PMs were isolated from WT mice, treated with IFN $\gamma$ or PBS (Ctrl), and ChIP assays were performed on the monocyte chemotactic protein 1 (MCP1) promoter $(-1,363$ to $-1,378)$ following immunoprecipitation with antiKLF6 or IgG (Ctrl). (h) RAW264.7 macrophages were transfected to overexpress the indicated plasmids, and then stimulated with IFN $\gamma$ or PBS (Control). Relative gene expression of MCP1 was determined by qPCR, and is expressed relative to PBS-treated, mock-transfected cells. Graphs show mean \pm s.e.m.; ${ }^{*} P \leqslant 0.05 ;{ }^{*} P \leqslant 0.005, n=3$. 
macrophages, which promote host defense by initiating immune responses against pathogens. Although M1 responses have evolved as a critical mechanism of host defense in response to pathogens, when left unchecked (as in the case of chronic inflammation, such as IBD), these responses can be extremely deleterious. The inflamed intestine is replete with proinflammatory cytokines, such as IFN $\gamma$, TNF, IL-1, and IL-6, which promote inflammation and tissue destruction, but also reinforce pro-inflammatory M1 macrophage activation. IBD patients produce high levels of pro-inflammatory cytokines and other soluble mediators, including nitric oxide, a byproduct of L-arginine, reactive oxygen species and nitric oxide synthases, cathepsins, and metalloproteinases. ${ }^{12}$ Thus, once inflammation is established, environmental cues provide reinforcement for pathogenic, M1 activation.

Mounting evidence suggests that the local microenvironment within the inflamed, IBD intestines can result in a dynamic shift in macrophage polarization away from an inflammatory-anergic phenotype and toward an M1 phenotype, thus significantly contributing to the pathogenesis of IBD. $^{35}$ It is therefore critical to identify the molecular mechanisms underlying cytokine-induced M1 activation in the setting of chronic intestinal inflammation in order to design more effective and targeted therapeutic approaches. Our observations of increased KLF6 mRNA in colon tissue from UC and CD patients, as well as significantly elevated KLF6 mRNA in acutely inflamed biopsy tissue from UC patients (Figure 1), suggest that locally high KLF6 levels correlate with worsened intestinal inflammation and tissue damage.

Given their role in directing lymphocyte phenotype and function, macrophages are at the epicenter of directing immune responses and are therefore an attractive target for therapeutic intervention. Pathogenic M1 polarization has been associated with numerous inflammatory conditions, including autoimmune diseases, atherosclerosis, and metabolic syndrome. Therefore, an enhanced understanding of the mechanisms by which macrophages are diverted away from the beneficial, protective M2 phenotypes will be relevant for many disease settings.

Transcriptional regulation is a central means of controlling macrophage activation. Several transcriptional regulators have been demonstrated to have a role in macrophage polarization, including NFKB, PPARs, and the STAT, AKT, and IRF (interferon regulatory factor) families. ${ }^{38}$ Previous studies have demonstrated that KLF2 and KLF4 have likewise been shown to regulate macrophage anti-inflammatory gene expression and function, although the role of these factors in macrophagemediated intestinal inflammation remains unknown. Interestingly, our data show that KLF6 functions in an opposing way to KLF2/4, by promoting pro-inflammatory M1 activation and diverting mucosal macrophages away from protective M2 activation. STAT1 and IRF5 also have demonstrated roles in promoting M1 phenotypes. $^{38,39}$ Therefore, the potential crossregulation of STAT1, IRF5, and KLF6 represents an important area for future study. Our data show that IFN $\gamma$ treatment of macrophages induces KLF6 expression (Figure 6,
Supplementary Figure S2), and IFN $\gamma$ has been shown to signal in both STAT1-dependent and -independent manners. ${ }^{40}$ Therefore, it is possible that STAT1 is upstream of KLF6, or that KLF6 is induced independently of STAT1.

Intriguingly, our studies show that regulation of macrophage activation by KLF6 occurs by two distinct pathways: cooperation with NFKB to induce pro-inflammatory gene expression, and inhibition of anti-inflammatory targets through STAT3 suppression (Figure 6). Thus, KLF6 appears capable of modulating pro- and anti-inflammatory transcriptional regulatory molecules, leading to diverse outcomes for target gene expression. One mechanism underlying macrophage inflammatory anergy is blockade of pro-inflammatory $\mathrm{NF \kappa B}$ signaling downstream of toll-like receptors, ${ }^{41}$ thus preventing $\mathrm{NF \kappa B}$ translocation to the nucleus and subsequent transcription of pro-inflammatory genes. Given our observations of elevated KLF6 in the myeloid cells and intestinal tissue of IBD patients, we hypothesize that high levels of KLF6 may act to overcome blockade in NFKB-mediated signaling, thereby contributing to macrophage escape from inflammatory anergy.

Alterations in KLF expression and/or function have been associated with the pathogenesis of numerous human diseases, including bacterial infection/sepsis, cardiovascular disease, metabolic disorders, and cancer. ${ }^{23,24}$ Interestingly, KLF6 has a known role as a tumor suppressor and was shown to be inactivated in the majority of spontaneous and IBD-associated colorectal cancer cases. ${ }^{42}$ This finding further highlights the importance of each particular tissue microenvironment in determining the ultimate function of gene regulatory proteins in different contexts. Because of its robust expression in myeloid cells and its intersection with numerous other transcriptional regulators, KLF6 represents an attractive candidate for targeted therapeutic approaches for IBD and other conditions mediated by inflammatory macrophages.

The present study is first to identify KLF6 as a strong inducer of pro-inflammatory M1 activation in the context of chronic intestinal inflammation. Monocyte-derived macrophages from IBD patients express significantly elevated levels of proinflammatory cytokines and diminished anti-inflammatory molecules, ${ }^{16}$ likely contributing to the inflammatory cytokine environment in the diseased tissue that reinforces M1 activation. Signaling downstream of KLF6 induces numerous pro-inflammatory gene targets, while simultaneously dampening the expression of anti-inflammatory targets; thereby, representing a central nexus point controlling many transcriptional targets. Thus, we propose that induction of KLF6, either in newly-recruited monocytes or in established tissue macrophages, participates in diverting macrophages away from protective, M2-like activation states and toward pathogenic, M1 activation. Given the central contribution of activated macrophages to chronic inflammation in IBD, this is likely an important mechanism leading to sustained tissue inflammation. Efforts at limiting the expression or functional activity of KLF6 represent promising areas for future development of biologics aimed at targeting innate immune mechanisms contributing to chronic intestinal inflammation. 


\section{METHODS}

Mice. Mac-KLF6-WT and Mac-KLF6-KO mice were generated by crossing Klf6 floxed mice with Lyz2cre mice expressing the lysozyme M promoter-driven Cre recombinase, as previously described. ${ }^{28}$ Mice harboring two Klf6 floxed alleles and two Lyz2cre alleles were designated Mac-KLF6-KO, and Lyz2cre mice (harboring only two Cre alleles) were designated Mac-KLF6-WT. Lyz2cre and Klf6 genotyping have been previously described. ${ }^{28}$ Mac-KLF6-KO mice display approximately $85-90 \%$ reduction in Klf6 expression in the myeloid compartment, as assessed by qPCR and western blot. ${ }^{28} \mathrm{TNF}^{\Delta \mathrm{ARE} /+}$ mice were generated by breeding male mice heterozygous for the $\mathrm{TNF}^{\triangle \mathrm{ARE}}$ mutant allele with $\mathrm{TNF}^{+/+}$females, which results in $50 \%$ of offspring with post-transcriptional dysregulation of TNF and spontaneous intestinal inflammation. $\mathrm{WT} \mathrm{TNF}^{+/+}$littermates were used as controls for these experiments. $\mathrm{TNF}^{\Delta \mathrm{ARE} /+}$ founder mice were originally provided by J. Rivera-Nieves (University of California, San Diego). ${ }^{31}$ All mice were bred and maintained under special pathogen free conditions, fed standard laboratory chow (Harlan Teklad, Indianapolis, IN), and kept on a 12-h light/dark cycle. All procedures were approved by the IACUC at Case Western Reserve University and conformed to guidelines established by the American Association for Accreditation of Laboratory Animal Care.

Human tissue samples. Colonic biopsies were obtained from healthy adult volunteers or patients with diagnosed CD or UC during endoscopy following informed consent. The control group consisted of individuals undergoing screening colonoscopy, who were included in the study only when endoscopy and pathology did not reveal any mucosal abnormalities. Endoscopies were performed at University Hospitals Case Medical Center, Cleveland, $\mathrm{OH}$ and at the Endoscopy Unit of the 1st Dept. of Internal Medicine, Propaedeutic, at Laikon Hospital, Athens, Greece. Specimens were maintained at $4{ }^{\circ} \mathrm{C}$ for $1-2$ days in RNAlater Solution (Ambion, Life Technologies, Austin, TX) until total RNA was extracted as described below. Peripheral blood mononuclear cells were prepared from heparinized peripheral blood by density centrifugation, and cell viability was determined by trypan blue exclusion. Monocytes were isolated from peripheral blood mononuclear cells by magnetic bead separation as described below. Informed consent was obtained from all human subjects under protocols approved by the Institutional Review Board of University Hospitals Case Medical Center/Case Western Reserve University. This study was conducted in compliance with good clinical practice and according to the principles of the Declaration of Helsinki.

Confocal immunofluorescent microscopy. 5- $\mu \mathrm{m}$ Tissue sections were cut from formalin-fixed, paraffin-embedded blocks. Sections were de-paraffinized and re-hydrated by sequential $4 \mathrm{~min}$ incubations in xylene and ethanol. Antigen retrieval was performed to unmask cross-linked epitopes by incubating sections for $20 \mathrm{~min}$ in boiling citrate buffer (Dako USA, Carpinteria, CA) and then cooling them to room temperature. Tissue was blocked with $10 \%$ chicken serum (Jackson ImmunoResearch, West Grove, PA) for $30 \mathrm{~min}$, and then labeled with mouse anti-mouse CD68 (Novus Biologicals, Littleton, CO), rabbit anti-mouse KLF6 (Santa Cruz Biotechnology, Dallas, TX), or appropriate isotype controls for $1 \mathrm{~h}$ at room temperature. Alexafluor 488- or 594-conjugated chicken anti-mouse or chicken anti-rabbit secondary antibodies (Life Technologies, Carlsbad, CA) were used to detect primary antibodies and Draq-5 (Axxora, San Diego, CA) was used as a nuclear marker. Images were collected using a $40 \times(\mathrm{NA}$ 0.85 ) objective on an UltraVIEW Vox spinning disk confocal system (Perkin Elmer, Waltham, MA) attached to a Leica DMI 6000B inverted microscope (Leica, Buffalo Grove, IL).

DSS colitis model. In all, 8- to 10-week-old male Mac-KLF6-WT and Mac-KLF6-KO mice were fed 3\% DSS (TdB Consultancy AB, Uppsala, Sweden) for 5 days ad libitum in their drinking water. Mice had access to standard laboratory chow throughout the experiment and were assessed on days 0 (initiation of DSS), 1, 3, and 5 for body weight and intestinal bleeding (hemoccult assay) by an investigator blinded to mouse genotype. Following 5 days of DSS, mice were subjected to colonic endoscopy and killed for collection of colon tissues.

Histologic evaluation of colon inflammation. Full-length colons of experimental mice were removed, flushed of fecal contents, opened longitudinally, and placed in Bouin's fixative for $24 \mathrm{~h}$. Tissues were embedded in paraffin, cut into $3 \mu \mathrm{m}$ sections and stained with $\mathrm{H} \& \mathrm{E}$. Disease severity was evaluated by a trained pathologist (Wei Xin, MD) in a blinded manner using an established histologic scoring system for colitis. ${ }^{33}$ Images were obtained on an Axiophot microscope, captured on an Axiocam and assembled using Axiovision Release 4.5 (Carl Zeiss, Thornwood, NY).

Endoscopic evaluation of murine colitis. Experimental colitis following DSS treatment was assessed by a validated, reproducible endoscopic scoring system encompassing visual assessment of the extent and severity of colitis, as well as a numeric scoring system. ${ }^{34}$ Briefly, following anesthetization with isoflurane, an Olympus URF-V flexible endoscope with attached camera (Olympus America, Center Valley, PA) was used to visualize transparency of the intestinal wall, mucosal bleeding, and focal lesions. Photos were acquired using narrow-band imaging to capture light of $390-445 \mathrm{~nm}$ and $530-550 \mathrm{~nm}$, highlighting structures containing hemoglobin. Perianal inflammation (diarrhea, bloody discharge, rectal prolapse, granulation, and fistulas) were observed before endoscopy procedure. A validated decimal-weighted scoring system ${ }^{34}$ was used to characterize endoscopic and perianal findings by a researcher blinded to mouse genotype. The system encompasses four parameters, each with a score of 0-3: perianal findings; wall transparency; intestinal bleeding; and focal lesions (edematous areas/erosions/ulcers).

Cell culture. RAW264.7 macrophages were cultured in DMEM (Dulbecco's modified Eagle medium) supplemented with 10\% fetal bovine serum, $100 \mathrm{U} \mathrm{ml}^{-1}$ penicillin, $10 \mu \mathrm{g} \mathrm{ml}^{-1}$ streptomycin, and $2 \mathrm{~mm}$ glutamine in a humidified incubator $\left(5 \% \mathrm{CO}_{2}\right.$ and $\left.37^{\circ} \mathrm{C}\right)$. Mouse PMs were obtained by lavage 3 days post injection with $3 \%$ thioglycolate broth in 8 - to 12 -week old mice, as previously described. ${ }^{26}$ Cells were cultured for short-term stimulation experiments in complete DMEM as described above. Mouse monocyte-derived macrophages were differentiated ex vivo by culturing $\mathrm{CD} 14^{+}$ monocytes (isolated by negative selection) in DMEM supplemented with recombinant mouse M-CSF-1 for 7 days, as previously described, ${ }^{28}$ and were then harvested and used for experiments. Human monocytes were isolated from unfractionated PBMCs by magnetic bead separation (Monocyte Isolation Kit 2, Miltenyi Biotec, San Diego, CA), and then cultured with recombinant human M-CSF $\left(50 \mathrm{ng} \mathrm{ml}^{-1}\right)$ ex vivo for 7 days to generate primary macrophages. All experiments involving human samples were approved by the Case Western Reserve University Institutional Review Board.

Transient transfection. Transfection of RAW264.7 cells was performed using Lipofectamine (Life Technologies, Carlsbad, CA) according to the manufacturer's instructions. Efficiency of transfection of either pCl-neo (EV control) or pCl-neo-KLF6 (over-expressing KLF6) averaged $65-75 \%$ in all experiments. Transfected cells were stimulated in vitro with IFN $\gamma\left(100 \mathrm{U} \mathrm{ml}^{-1}\right), \mathrm{IL}-10\left(10 \mathrm{ng} \mathrm{ml}^{-1}\right)$, or PBS (control), then harvested and used for experiments.

Luciferase assays. RAW264.7 cells were transfected using the Lipofectamine reagent (Life Technologies) according to the manufacturer's instructions. Luciferase reporter plasmids driven by the NFKB concatemer (Promega, Madison, WI) or STAT3 (Affymetrix, Santa Clara, CA) were transfected alone or together with plasmids encoding KLF6 or siKLF6. Cells were treated with IFN $\gamma$ $\left(100 \mathrm{U} \mathrm{ml}^{-1}\right)$ or IL-10 $\left(10 \mathrm{ng} \mathrm{ml}^{-1}\right)$ for $16 \mathrm{~h}$, followed by measuring Luciferase reporter activity. Data were normalized relative to the control (PBS-treated) group. 
Chromatin immunoprecipitation. ChIP experiments were performed using the EZ-Magna ChIP G kit (Millipore, Billerica, MA) according to the manufacturer's instructions. Briefly, PMs from WT mice were isolated and stimulated ex vivo with IFN $\gamma\left(100 \mathrm{U} \mathrm{ml}^{-1}\right)$, IL-10 $\left(10 \mathrm{ng} \mathrm{ml}^{-1}\right)$, or PBS (control). Chromatin immunoprecipitations were performed using anti-KLF6 antibody (Santa Cruz Biotechnology) or IgG. Precipitated fragments were analyzed by real-time qPCR using primer sequences flanking the MCP1 promoter $(-1,363$ to $-1,378)$ or SOCS3 promoter $(-313$ to -319$)$.

Western blot. Western blots for KLF6 were performed using total cell lysates. Primary antibody against KLF6 (Santa Cruz Biotechnology) was used at a 1:1,000 dilution, and polyclonal HRP-conjugated goat anti-rabbit IgG at 1:5,000 dilution (Millipore) was used as a secondary antibody.

RNA extraction, reverse-transcription, and real-time quantitative PCR assay. Total RNA was isolated from tissue samples using Trizol (Life Technologies) or from macrophages using the High Pure RNA Isolation Kit (Roche Life Science, Indianapolis, IN). One microgram of total RNA was reverse-transcribed using M-MuLV reverse transcriptase (New England Biolabs, Ipswich, MA) in the presence of random hexamers and oligo(dT) primers. Real-time quantitative PCR was performed using University SYBR Green PCR Master Mix on Applied Biosystems Step One Plus real-time PCR system (Applied Biosystems, Grand Island, NY) using gene-specific primers.

Statistical analysis. Data are presented as mean \pm s.e.m. unless otherwise indicated. Two-tailed Student's T-tests with Bonferroni's corrections were performed to assess the significance of differences between groups. $P$ values $<0.05$ were considered as significant.

SUPPLEMENTARY MATERIAL is linked to the online version of the paper at http://www.nature.com/mi

\section{ACKNOWLEDGMENTS}

We thank Scott Howell, Minh Lam, Xiao-Ming Wang, and Becky Sebastian for their technical support and assistance. This work was supported by grants from the NIH: HL097023, HL121193, and HL126626 (to GHM), DK056762 (to TTP), DK091222 and DK097948 (to TTP/FC), K01DK105138 (to WAG) and from the Crohn's and Colitis Foundation of America: CDA329284 (to WAG) and RFA326877 (to CDS).

\section{AUTHOR CONTRIBUTIONS:}

G.H.M., T.T.P., and W.A.G. conceived and designed the study. G.H.M., W.A.G., S.O., D.D., G.D.K., L.D.M., and C.D.S. performed experiments. G.H.M., T.T.P., F.C., W.A.G., S.O., D.D., and L.D.M. analyzed and interpreted the data. G.B. and S.C. collected and provided human specimens. W.A.G., G.H.M., and T.T.P. wrote and edited the manuscript that is approved by all authors.

\section{DISCLOSURE}

The authors declared no conflict of interest.

(c) 2016 Society for Mucosal Immunology

\section{REFERENCES}

1. Round, J.L. \& Mazmanian, S.K. The gut microbiota shapes intestinal immune responses during health and disease. Nat. Rev. Immunol. 9, 313-323 (2009).

2. Lee, S.H., Starkey, P.M. \& Gordon, S. Quantitative analysis of total macrophage content in adult mouse tissues. Immunochemical studies with monoclonal antibody F4/80. J. Exp. Med. 161, 475-489 (1985).

3. Smith, P.D. et al. Intestinal macrophages and response to microbial encroachment. Mucosal Immunol. 4, 31-42 (2011).

4. Qualls, J.E., Kaplan, A.M., van Rooijen, N. \& Cohen, D.A. Suppression of experimental colitis by intestinal mononuclear phagocytes. J. Leukoc. Biol. 80, 802-815 (2006).
5. Hunter, M.M. et al. In vitro-derived alternatively activated macrophages reduce colonic inflammation in mice. Gastroenterology 138, 1395-1405 (2010).

6. Rakoff-Nahoum, S., Paglino, J., Eslami-Varzaneh, F., Edberg, S. \& Medzhitov, R. Recognition of commensal microflora by toll-like receptors is required for intestinal homeostasis. Cell 118, 229-241 (2004).

7. Lin, H.H. et al. The macrophage $\mathrm{F} 4 / 80$ receptor is required for the induction of antigen-specific efferent regulatory Tcells in peripheral tolerance. J. Exp. Med. 201, 1615-1625 (2005).

8. Iwasaki, A. \& Medzhitov, R. Control of adaptive immunity by the innate immune system. Nat. Immunol. 16, 343-353 (2015).

9. Janeway, C.A. Jr. Approaching the asymptote? Evolution and revolution in immunology. Cold Spring Harb. Symp. Quant. Biol. 54, 1-13 (1989).

10. Murray, P.J. et al. Macrophage activation and polarization: nomenclature and experimental guidelines. Immunity 41, 14-20 (2014).

11. Smythies, L.E. et al. Human intestinal macrophages display profound inflammatory anergy despite avid phagocytic and bacteriocidal activity. J. Clin. Invest. 115, 66-75 (2005).

12. Mowat, A.M. \& Bain, C.C. Mucosal macrophages in intestinal homeostasis and inflammation. J. Innate Immun. 3, 550-564 (2011).

13. Li, B., Alli, R., Vogel, P. \& Geiger, T.L. IL-10 modulates DSS-induced colitis through a macrophage-ROS-NO axis. Mucosal Immunol. 7, 869-878 (2014).

14. Takeda, K. et al. Enhanced Th1 activity and development of chronic enterocolitis in mice devoid of Stat3 in macrophages and neutrophils. Immunity 10, 39-49 (1999).

15. Hontecillas, R. et al. Immunoregulatory mechanisms of macrophage PPAR-gamma in mice with experimental inflammatory bowel disease. Mucosal Immunol. 4, 304-313 (2011).

16. Shouval, D.S. et al. Interleukin-10 receptor signaling in innate immune cells regulates mucosal immune tolerance and anti-inflammatory macrophage function. Immunity 40, 706-719 (2014).

17. Dijkstra, G. et al. Increased expression of inducible nitric oxide synthase in circulating monocytes from patients with active inflammatory bowel disease. Scand. J. Gastroenterol. 37, 546-554 (2002).

18. Hausmann, M. et al. Cathepsin $D$ is up-regulated in inflammatory bowel disease macrophages. Clin. Exp. Immunol. 136, 157-167 (2004).

19. Reinecker, H.C. et al. Enhanced secretion of tumour necrosis factor-alpha, IL-6, and IL-1 beta by isolated lamina propria mononuclear cells from patients with ulcerative colitis and Crohn's disease. Clin. Exp. Immunol. 94, 174-181 (1993).

20. Targan, S.R. et al. A short-term study of chimeric monoclonal antibody CA2 to tumor necrosis factor alpha for Crohn's disease. Crohn's Disease cA2 Study Group. N. Engl. J. Med. 337, 1029-1035 (1997).

21. Watanabe, N. et al. Elimination of local macrophages in intestine prevents chronic colitis in interleukin-10-deficient mice. Dig. Dis. Sci. 48, 408-414 (2003).

22. Scheinin, T., Butler, D.M., Salway, F., Scallon, B. \& Feldmann, M. Validation of the interleukin-10 knockout mouse model of colitis: antitumour necrosis factor-antibodies suppress the progression of colitis. Clin. Exp. Immunol. 133, 38-43 (2003).

23. Cao, Z., Sun, X., Icli, B., Wara, A.K. \& Feinberg, M.W. Role of Kruppel-like factors in leukocyte development, function, and disease. Blood 116, 4404-4414 (2010).

24. McConnell, B.B. \& Yang, V.W. Mammalian Kruppel-like factors in health and diseases. Physiol. Rev. 90, 1337-1381 (2010).

25. Pearson, R., Fleetwood, J., Eaton, S., Crossley, M. \& Bao, S. Kruppel-like transcription factors: a functional family. Int. J. Biochem. Cell Biol. 40, 1996-2001 (2008).

26. Mahabeleshwar, G.H. et al. The myeloid transcription factor KLF2 regulates the host response to polymicrobial infection and endotoxic shock. Immunity 34, 715-728 (2011).

27. Sharma, N. et al. Myeloid Kruppel-like factor 4 deficiency augments atherogenesis in ApoE-/- mice-brief report. Arterioscler. Thromb. Vasc. Biol. 32, 2836-2838 (2012).

28. Date, D. et al. Kruppel-like transcription factor 6 regulates inflammatory macrophage polarization. J. Biol. Chem. 289, 10318-10329 (2014).

29. Palau, N. et al. Genome-wide transcriptional analysis of T cell activation reveals differential gene expression associated with psoriasis. BMC Genomics 14, 825 (2013). 
30. Bechmann, L.P. et al. Post-transcriptional activation of PPAR alpha by KLF6 in hepatic steatosis. J. Hepatol. 58, 1000-1006 (2013).

31. Ho, J. et al. A CD8 +/CD103high T cell subset regulates TNF-mediated chronic murine ileitis. J. Immunol. 180, 2573-2580 (2008).

32. Okayasu, I. et al. A novel method in the induction of reliable experimental acute and chronic ulcerative colitis in mice. Gastroenterology 98, 694-702 (1990).

33. Corridoni, D. et al. Dysregulated NOD2 predisposes SAMP1/YitFc mice to chronic intestinal inflammation. Proc. Natl. Acad. Sci. USA 110, 16999-17004 (2013)

34. Kodani, T. et al. Flexible colonoscopy in mice to evaluate the severity of colitis and colorectal tumors using a validated endoscopic scoring system. J. Vis. Exp 16, e50843 (2013).

35. Bain, C.C. \& Mowat, A.M. Macrophages in intestinal homeostasis and inflammation. Immunol. Rev. 260, 102-117 (2014).

36. O'Farrell, A.M., Liu, Y., Moore, K.W. \& Mui, A.L. IL-10 inhibits macrophage activation and proliferation by distinct signaling mechanisms: evidence for
Stat3-dependent and -independent pathways. EMBO J. 17, 1006-1018 (1998).

37. Mills, C.D., Lenz, L.L. \& Ley, K. Macrophages at the fork in the road to health or disease. Front. Immunol. 6, 59 (2015).

38. Lawrence, T. \& Natoli, G. Transcriptional regulation of macrophage polarization: enabling diversity with identity. Nat. Rev. Immunol. 11, 750-761 (2011).

39. Ostuni, R. \& Natoli, G. Transcriptional control of macrophage diversity and specialization. Eur. J. Immunol. 41, 2486-2490 (2011).

40. Ramana, C.V., Gil, M.P., Schreiber, R.D. \& Stark, G.R. Stat1-dependent and -independent pathways in IFN-gamma-dependent signaling. Trends Immunol. 23, 96-101 (2002).

41. Smythies, L.E. et al. Inflammation anergy in human intestinal macrophages is due to Smad-induced IkappaBalpha expression and NF-kappaB inactivation. J. Biol. Chem. 285, 19593-19604 (2010).

42. Reeves, H.L. et al. Kruppel-like factor 6 (KLF6) is a tumor-suppressor gene frequently inactivated in colorectal cancer. Gastroenterology 126, 1090-1103 (2004). 